\title{
Complicaciones del parto vertical en el Instituto Nacional Materno Perinatal de Lima, Perú. Enero a julio del 2012
}

\author{
Marco Castillo Ayarza', Alejandra Cahuata Mosqueira', Alejandro Calle Brush', Ricardo Muñoz Silva²
}

\section{RESUMEN}

Objetivo: Determinar las complicaciones más frecuentes durante la atención del parto vertical.

Material y Métodos: Se revisaron las historias clínicas de 85 madres que fueron atendidas por parto vertical en el Instituto Nacional Materno Perinatal.

Resultados: no hubo complicación en 11 casos (9\%). En 52 (40\%) de los casos se tuvo que realizar una episiotomía. El desgarro vaginal ocurrió en 25 pacientes (19\%). Hubo presencia de líquido meconial en 13 pacientes (10\%). En 7 casos (6\%) hubo alumbramiento incompleto, que requirió legrado uterino. La hemorragia ocurrió en 6 pacientes (5\%). Respecto a desgarro cervical, trabajo de parto prolongado y trabajo de parto precipitado, cada uno ocurrió en 4 pacientes (3\%). Se reportó atonía uterina en 3 pacientes (2\%). Excluyendo la episiotomía como complicación, la frecuencia de complicaciones disminuiría de $89 \%$ a $48 \%$.

Conclusión: Las complicaciones fueron semejantes a la atención horizontal. No se observó efecto nocivo del parto en posición vertical. Se recomienda estudiar muestras más grandes con registro de datos diferenciados que permita conocer la variable: personal de salud. (Horiz Med 2014; 14(1): 31-37)

Palabras clave: Parto vertical, complicaciones del parto. (Fuente: DeCS BIREME).

Delivery complications in patients with vertical position during childbirth at a Maternity Hospital in Lima, Peru. January - June 2012

\section{ABSTRACT}

Objective: To determine the most frequent complications during vertical childbirth.

Material and Methods: 85 medical histories of vertical childbirth during labor were reviewed. Within the sample of 85 patients

Results: 11 cases (9\%) showed no complications. In 52 patients (40\%) episiotomy was performed. Vaginal tearing occurred in 25 patients (19\%). Liquid meconium was present in 13 patients (10\%). In 7 cases (6\%) there was an incomplete delivery, which had to be supplemented with curettage. Hemorrhaging occurred in 6 patients (5\%). Cervical laceration, prolonged labor and precipitated labor all occurred in 4 patients (3\%). Uterine atony was reported in 3 patients (2\%). Excluding episiotomy as a complication, the complication rate would fall from $89 \%$ to $48 \%$.

Conclusion: Complications were similar to the horizontal attention. No adverse effect was observed delivery upright. It is recommended to study larger samples with different data log variable that allows to know: health provider. (Horiz Med 2014; 14(1): 31-37)

Key words: Vertical delivery, delivery complications. (Source: MeSH NLM).

\footnotetext{
${ }^{1}$ Estudiantes de Sexto Año de la FMH - USMP.

${ }^{2}$ Médico, Ginecólogo Obstetra del Instituto Nacional Materno Perinatal. Lima, Perú
} 


\section{INTRODUCCIÓN}

El parto vertical, se refiere a la posición de la gestante en posición vertical, ya sea sentada, apoyándose en una o dos rodillas, cogida de sogas, 0 en cuclillas. El personal que atiende el parto debe colocarse adelante 0 detrás de la gestante (1).

Históricamente, el parto vertical ha sido documentado por grabados y esculturas como un método de parto utilizado por varias culturas alrededor del mundo (2).

Desde 1996, la OMS ha rectificado la seguridad del parto vertical y ha recomendado que el tipo de parto que se practique dependa en las preferencias de la madre (3).

En el Perú, las prácticas del parto vertical fueron institucionalizadas en el 2005, por el Ministerio de Salud ("Estrategia Sanitaria de Salud Sexual y Reproductiva. Norma Técnica para la atención del parto vertical") (4).

Desde el 2007 hasta el 2013, se informó que más de 1,200 partos verticales se atendieron en el Instituto Nacional Materno Perinatal (INMP) (5).

Las indicaciones para el parto vertical son similares a cualquier parto natural: no complicaciones obstétricas, presentación cefálica, no incompatibilidad pélvico-fetal.

Del mismo modo, sus contraindicaciones: cesárea anterior, incompatibilidad pélvico fetal, sufrimiento fetal, feto en podálico, embarazo gemelar, distocias, prematuridad, hemorragia del tercer trimestre, ruptura prematura de membranas, preeclampsia severa, antecedente de parto complicado y embarazo pos término.

Se han atribuido beneficios fisiológicos al parto vertical. En esta posición, el útero de la gestante no comprime los grandes vasos, y así no ocasiona alteraciones en la circulación materna y placentaria, mejorando la oxigenación del feto (6).

La posición permite que las fuerzas de gravedad ayuden al encajamiento y descenso del feto (7) y también favorece la acomodación pélvico fetal, ampliando $2 \mathrm{~cm}$ el diámetro anteroposterior y $1 \mathrm{~cm}$ el diámetro transverso (8).

Existe un mej or equilibrio ácido base fetal y mej ora la ventilación pulmonar de la madre (9).

Las diferentes posiciones verticales permiten que los miembros inferiores sirvan como un punto de apoyo y así ayudan indirectamente a la prensa abdominal para la salida del feto (10).

El parto vertical también proporciona beneficio psicoafectivo, con un mayor rol protagónico en la decisión de su vía de parto y una mayor participación en el nacimiento de su hijo (11).

Según la norma técnica del MINSA, se pueden presentar complicaciones: mayor sangrado cuando no se utiliza el alumbramiento dirigido, laceraciones del periné, salida brusca del feto, prolapso del cordón, procidencia de miembros superiores, distocia de hombros.

El 2008, se publicó (Rev Per Ginecol y Obst), el trabajo "Parto vertical: Retornando a una costumbre ancestral" que compara las ventajas del parto vertical y el parto horizontal, se observó que en el parto vertical el periodo expulsivo fue menor, pero ocasionó más sangrado que el parto horizontal, además de mayor número de desgarros.

La percepción de satisfacción y comodidad con el parto fue de $96 \%$, comparado con el $46 \%$ del parto horizontal.

Otros estudios también confirman que a pesar a de los beneficios y seguridad del parto vertical esta puede estar asociada a complicaciones importantes (12-19).

A pesar que la práctica del parto vertical ha sido institucionalizada mediante guías y sus ventaj as son expuestas en medios de comunicación al público en general, consideramos que no existe suficiente información sobre esta forma de atención.

Por lo cual, el objetivo del trabajo fue determinar las complicaciones más frecuentes que ocurren durante la atención del parto vertical. 


\section{MATERIAL Y MÉTODOS}

Estudio de tipo observacional, descriptivo, transversal, cuantitativo, retrospectivo.

Se revisaron las Historias Clínicas de 85 pacientes que tuvieronparto vertical en el Instituto Nacional Materno Perinatal entre enero y julio del 2012.Se consideró como complicación:

- Hemorragia: Pérdida sanguínea mayor a 500 cc.

- Realización de episiotomía.

- Desgarro cervical.

- Desgarro vaginal.

- Atonía uterina.

- $\quad$ Alumbramiento Incompleto.

- $\quad$ Presencia de líquido meconial.

- $\quad$ Parto prolongado: Trabajo de parto de duración mayor a 12 horas.

- $\quad$ Parto precipitado: Trabajo de parto de duración menor a 03 (tres) horas.

\section{RESULTADOS}

No hubo ninguna complicación en 11 casos (9\%). En $52(40 \%)$ se realizó episiotomía. El desgarro vaginal ocurrió en 25 pacientes (19\%).

Hubo presencia de líquido meconial, indicativo de sufrimiento fetal agudo, en 13 pacientes (10\%). En 7 casos $(6 \%)$ hubo alumbramiento incompleto, el cual tuvo que complementarse con un legrado uterino.

La hemorragia (pérdida sanguínea mayor a $500 \mathrm{cc}$ ) ocurrió en 6 pacientes (5\%). Respecto a desgarro cervical, trabajo de parto prolongado (duración mayor a 12 horas) y trabajo de parto precipitado (menor a 3 horas), cada uno ocurrió en 4 pacientes $(3 \%)$.

Como complicación de menor incidencia se reportó la atonía uterina en 3 pacientes (2\%) (Figuras 1 y 2 ).

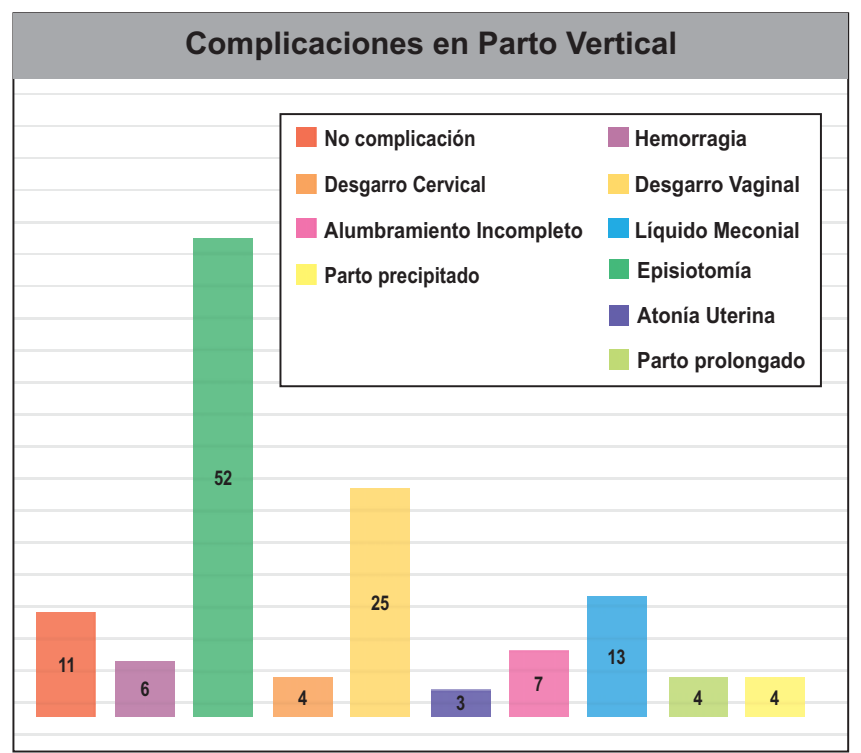

Figura 1. Complicaciones registradas en la revisión de Partos Verticales.

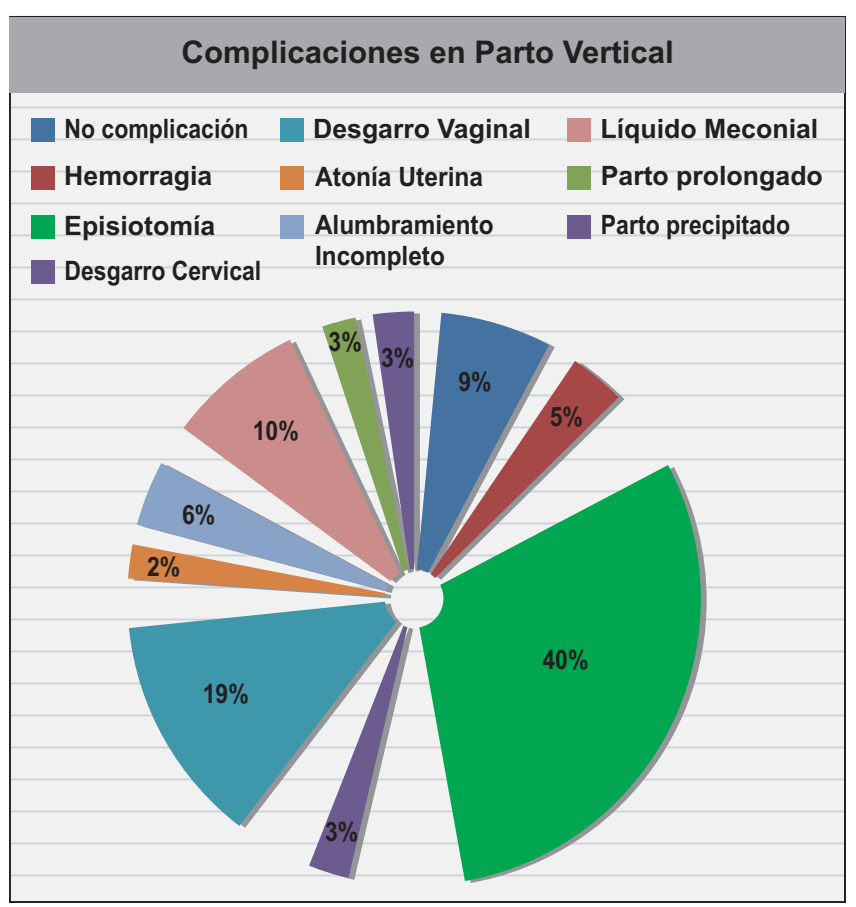

Figura 2. Complicaciones registradas en la revisión de Partos Verticales (en porcentajes).

Si consideramos sólo a la Episciotomía, y no se considera como una complicación, la ausencia de éstas aumenta de $9 \%$ a $52 \%$, mientras que la frecuencia de complicaciones disminuye de $89 \%$ a $48 \%$ (Figuras 3 y 4 ). 


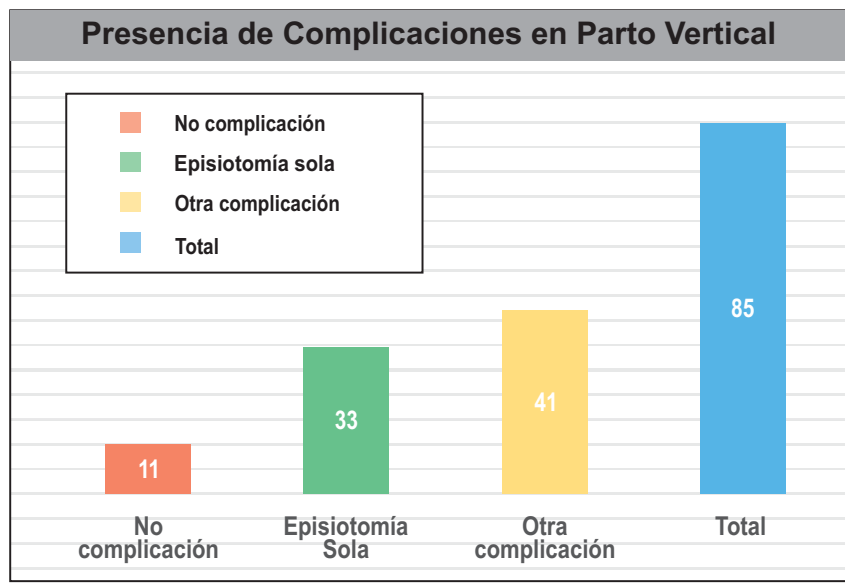

Figura 3. Presencia de Complicaciones en Parto Vertical excluyendo Episiotomías solas

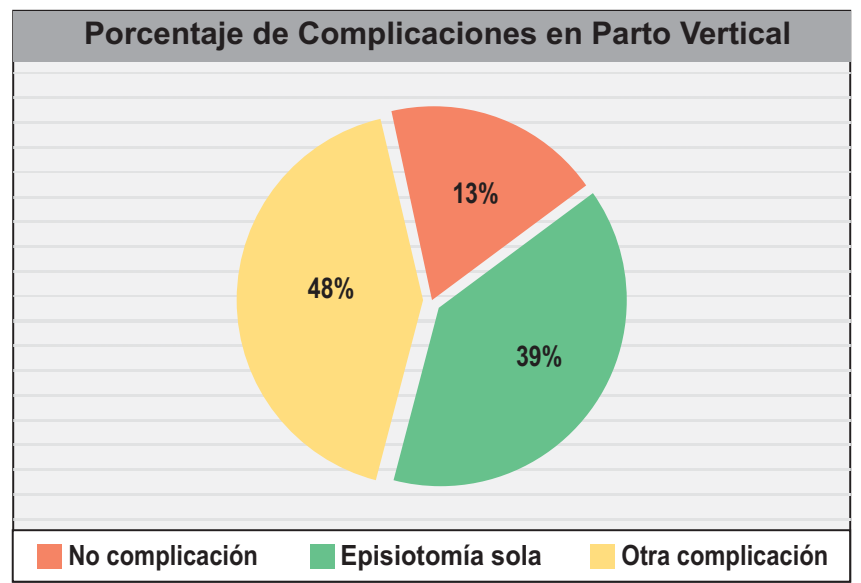

Figura 4. Porcentaje de Complicaciones en Parto Vertical excluyendo solo Episiotomías.

\section{DISCUSIÓN}

La atención humanizada de la madre durante el parto, de acuerdo con sus comodidades y su libertad de elección son temas de actualidad en nuestro país (15) y en los países latinoamericanos, pues existe variabilidad cultural donde se hace imperativo integrar a las costumbres ancestrales con el manejo institucionalizado, para adecuar los cuidados de salud a toda la población, tener pacientes más satisfechas y dispuestas a acudir a centros de salud a recibir cuidados por personal entrenado, disminuyendo riesgos y mortalidad.

Nuestro país es uno de los lugares pioneros en la atención del parto vertical, cuyas iniciativas han sido seguidas y puestas en práctica por países como Ecuador, México, Argentina, Colombia, entre otros (16).

Se han desarrollado Normas que promueven la atención de la gestante respetando las posiciones que desee adoptar, siempre que no exista contraindicación médica (17).

De la aplicación de estas Normas, surge la necesidad de observar, cuantificar y realizar estudios sobre la atención del parto vertical.

Cabe resaltar que nuestro trabaj o fue de naturaleza descriptiva, solo considerando las complicaciones registradas en las historias clínicas de una muestra de pacientes atendidas en parto vertical, no se comparó con pacientes atendidas en posición horizontal como en la literatura consultada.

En otros estudios, se evaluaron las variables de nuestro trabajo, además del dolor en el segundo periodo de trabajo de parto y puerperio inmediato, necesidad de instrumentar el parto, comodidad en la postura y resultados perinatales. También se evaluó la necesidad de cesárea (19).

No nos fue posible evaluar el dolor en el segundo periodo de trabajo de parto y puerperio inmediato pues este dato no figura en las historias clínicas.

Es importante resaltar el número de pacientes sin complicaciones, siendo éstos el 52\% (44 pacientes), y sin complicaciones ni necesidad de episiotomía, un total de 11 (13\%).

Algunos estudios sostienen que la postura vertical es factor de riesgo de desgarros vaginales y cervicales (19). Nuestros resultados guardan relación con esta afirmación, aunque no podemos afirmar que sea un factor de riesgo comparado con el parto horizontal.

Sin embargo, otros estudios realizados en el Perú, evaluando muestras similares de partos verticales (80 pacientes) no registraron mayor frecuencia de desgarros en el parto vertical (20).

Sobre la necesidad de episiotomía que en nuestro trabajo fue de 52 pacientes (40\%), esto al parecer dependió de la decisión del que atiende el parto. 
La literatura no ha demostrado disminuir la incidencia de distopia, incontinencia urinaria, desgarros mayores ni hemorragias y que más bien, se la asocia auna mayor incidencia de desgarros profundos (tercer y cuarto grados) frente al parto sin episiotomía (21).

Considerando este dato, sería beneficioso el parto vertical, pues varios estudios han descrito que en éste hay significativamente menor necesidad de esta técnica $(12,20)$, sin que eso signifique que haya más desgarros profundos.

En relación con la pérdida sanguínea, es importante señalar que el diagnóstico de pérdida sanguínea mayor de $500 \mathrm{ml}$, particularmente en el parto vertical debe ser interpretado con precaución, en especial porque la estimación de las pérdidas es influenciada por el observador, debido a que la sangre se recolecta en un receptáculo. Haciendo esta acotación, muchos estudios relacionan el parto vertical con mayor pérdida sanguínea (22), mientras que en nuestro estudio se observó que en sólo seis pacientes se registró una pérdida mayor de $500 \mathrm{ml}$. Sobre este tema, en la literatura hay referencias distintas.

Hay revisiones que señalan que el volumen de sangrado fue menor en forma global para el grupo de parto vertical, pero que esto se relaciona con el manejo activo del alumbramiento (lo cual disminuye el sangrado en relación al manejo tradicional) $(20,23)$.

En estudios que toman en cuenta la opinión subj etiva de las madres puérperas los aspectos positivos de la posición vertical emergieron de forma más intensa y frecuente que los negativos.

En general se percibe que es una posición más cómoda y que facilita la expulsión del feto. El parto horizontal, se ha relacionado con una gestante más tranquila al asumir, un papel más pasivo $(24,25)$.

Un punto interesante con respecto al dolor sería relacionar la posición de partos y la necesidad o uso de anestesia epidural (26).

Con respecto a la duración del parto, nuestro trabajo solo describe a los registrados como partos precipitadosy partos prolongados, pero hay estudios que relacionan el parto vertical con la aceleración de la primera fase del trabajo de parto (8). Con acortamiento del expulsivo en 8 minutos (27) o en 4.3 minutos (12).

En cuanto a las maniobras utilizadas en el trabajo de parto, éstas no fueron descritas en las historias que consultamos, pero es interesante notar que en trabajos anteriores se observó que maniobras como las de Kristeller, no son necesarias durante la atención del parto en posición vertical (28).

En conclusión, no hubo diferencia significativa en las complicaciones.

Con la posible excepción del nivel de pérdida sanguínea, no se han demostrado efectos nocivos del parto en posición vertical para la madre o el feto.

Debería abrirse una variable en las historias para registrar las características de un parto vertical, diferenciando la del parto vaginal horizontal a fin de realizar mayores evaluaciones con respecto a los beneficios y complicaciones frente al parto horizontal.

\section{AGRADECIMIENTOS}

Al Instituto Nacional Materno Perinatal, por brindarnos las facilidades para la investigación. A la Docente Guigliana Ayllón por su apoyo.

\section{Fuente de Financiamiento}

El presente trabajo fue financiado a través de recursos propios de los investigadores.

\section{Conflictos de interés}

Los autores declaran no tener conflictos de interés en la publicación de este artículo. 


\section{REFERENCIAS BIBLIOGRÁFICAS}

1. Ministerio de Salud. Dirección General de Salud de las personas. Estrategia Sanitaria de Salud Sexual y Reproductiva. Norma Técnica para la atención del parto vertical. 2005

2. JarchoJ.Postures\&practicesduringlaboramong primitive peoples. New York: Paul Hoeber, 1934.

3. OMS Asistencia en el parto normal: una guía práctica. Ginebra, Organización Mundial de Salud; 1996.

4. RPP. Ventajas del Parto Verticalenel Perú. Agosto 2010 [Internet] [Citado el 08/04/13. Disponible en http:// www.rpp.com. pe/201008-21-ventajas-del-parto-vertical-en-el-perunoticia_289377.html]

5. RPP Más de 1.200 partos verticales atendió la Maternidad de Lima. Marzo 2013. 2010 [Internet] [Citado el 08/04/13.Disponible en http:/ / www.rpp.com. pe/ 2013-03-07-mas-de1-200-partos-verticales-atendio-Iamaternidad-de-lima noticia_573742.html]

6. Bieniarz J, Crottogini JJ, Curuchet E, Romero G, Yoshida T, Poseiro JJ, et al. Aortocaval compression by the uterus in the late human pregnancy. II.

An arterio graphic study. Am J Obstet Gynecol 1968;100:203-17.

7. Sabatini H. Parto fisiológico en la posición vertical es la fisiológica para el parto. Universidad de Campinas. Sao Paulo, Brasil; 1992

8. Arroyo J y col. La posición de pie durante el trabajo de parto espontáneo. I. Efectos sobre la contractibilidad uterina. Dolor y duración del parto. Clin Invest Obstet Ginecol 1994;1:221.

9. Caldeyro-Barcia R, Giussi G, Storch E, Poseyro JJ, et al. The bearing- down efforts and their effects on fetal heart rate, oxygenation and acid-base balance. J Perinat Med 1981:9:63-7
10. Cuerva A, Márquez S. Consejería de Salud Agencia de Evaluación de Tecnologías Sanitarias de Andalucía. Fase Expulsiva del Parto: comparación entre la posición de la mujer, vertical frente a la horizontal, a través de los resultados maternos y fetales.

Informe 13-2006

11. Page L. The humanization of birth. Intern J Gynaecol Obstet 2001;75:S55-S58

12. Gupta JK, Hofmeyr GJ, Smyth R. Position in the second stage of labour for women without epidural anaesthesia. Cochrane Database of Systematic Reviews 2004

13. Crowley P, Elbourne D, Ashurst H, Murphy D, Duigan N. Delivery in an obstetric birth chair: a randomisedcontrolled trial. $\mathrm{Br} J$ Obstet Gynaecol 1991; 98(7):667-74

14. Babayer M, Bodack MP, Creatura C. Common peroneal neuropathy secondary to squatting during childbirth. Obstet Gynecol 1998, 91:830

15. Guibovich, A. Adecuación cultural al parto: Estudio comparativo del parto en posición vertical vs horizontal en el HN Arzobispo Loayza. Boletin Científico ASPPO 2012; 14(40): 3-5.

16. The state of the world's midwifery. 2011 Parto vertical en el Perú: contribuye a mejorar la salud y reducir la mortalidad materna. Junio 2011. [Internet]. URL:http:// www. unfpa.org/ sowmy/ resources/docs/ b a ckground_papers / $45_{-}$Colegio ObstetrasPeru_Parto Vertical.PDF

17. Gobierno de México. Secretaría de Salud. Dirección general de planeación y desarrollo en salud. La posición tradicional de atención del parto en los servicios de salud. 2007

18. Ministerio de Salud. Instituto Nacional Materno Perinatal. Guías de práctica clínica y de procedimientos en obstetricia y perinatología 2010. 
19. Calvo O, Flores AL, Morales VE. Comparación de resultados obstétricos y perinatales del parto en postura vertical versus supina. Ginecol Obstet Mex 2013;81:1-10.

20. Calderón J, Bravo J, et al. Parto vertical: retornando a una costumbre ancestral. Rev Per Ginecol Obstet 2008;la54:49-57

21. Gareberg B, Magnusson B, Sultan B, et al. Birth in standing position: $A$ high frequency of third degree tears. ActaObstetGynecolScand1994;73:630-3.

22. Martínez-Galiano JM. Prevención de las hemorragias posparto con el manej 0 activo del alumbramiento. MatronasProf 2009;10(4):20-26

23. Edianez $M$, Bruggemann $M$. Percepciones de puérperas sobre la vivencia durante el parto en la posición vertical y horizontal Rev Latino-am Enfermagem 2009 março-abril; 17(2)

24. Lugones $M$, Ramirez $M$. El parto en diferentes posiciones a través de la ciencia, la historia y la cultura. Revista Cubana de Ginecología y Obstetricia 2012;38(1):134-145

25. UNICEF. PARTO VERTICAL: El derecho de las mujeres a dar a luz de pie. [Internet]. URL:http: / / www. unicef.org/ peru/ spanish/ parto_vertical.pdf S.
26. Saavedra. Servicios de Salud Araucaria IX Región. Ministerio de Salud de Chile. Primer Encuentro Nacional Salud y Pueblos Indígenas. OPS-OMS. Chile 1996.

27. Terry RR, Westcott J, O'Shea L, Kelly F. Post partum out comes in supine delivery by physicians vs non supine delivery by midwives. JAOA 2006 April; 106(4):199-202.

28. Waldenström U, Gottvall K. A ramdomized controlled trial of birthing stool or conventional semi-recumbent position for second stage of labor. Birth 1991;18:5-10.

\section{Correspondencia:}

Alejandra Berenice Cahuata Mosqueira Dirección: Calle Tangarara , F:30 Urb. La Capullana, Santiago de Surco, Lima-Perú

Teléfono: 943576001.

Correo electrónico: alemb_7@hotmail.com 